\title{
A Petrov-Galerkin Finite Element Scheme for 1-D Time-independent Hamilton-Jacobi-Bellman Equations
}

\author{
(時間非依存の1次元ハミルトン・ヤコビ・ベルマン方程式に対する \\ ペトロフ・ガレルキン有限要素スキーム)

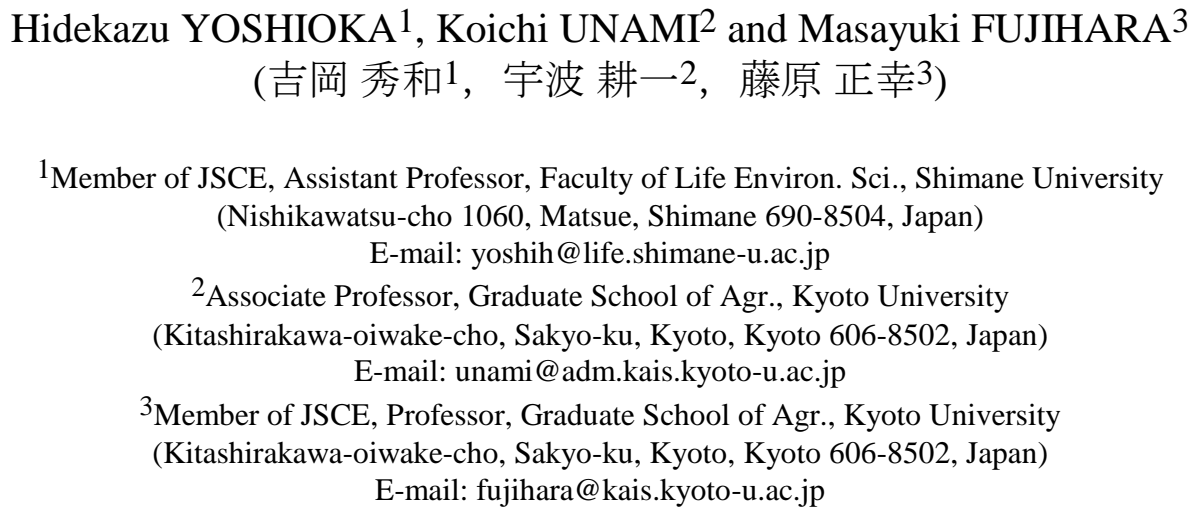

A numerical method for solving 1-D time-independent Hamilton-Jacobi-Bellman equations, which are referred to as 1-D HJBEs, is presented and applied to test cases for assessing its computational performance. An HJBE in this paper is a nonconservative second-order ordinary differential equation having linear diffusion and nonlinear drift terms. This paper applies a regularization method to the drift coefficient of the HJBEs, which helps well-pose the boundary value problems of the equations in the classical sense. A mathematical analysis on consistency errors between the solutions to the original and regularized HJBEs is performed. The derived results of the analysis show that the regularization method is mathematically consistent. The regularized HJBEs are solved with a Petrov-Galerkin finite element scheme, which is referred to as the PGFE scheme. The scheme is based on the fitting technique and is unconditionally stable for linear problems. Application of the scheme with the regularization method to the HJBEs with bounded drift coefficients demonstrates its satisfactory high computational accuracy. The optimal regularization parameter value as a function of the element size is then numerically identified. The computational results show that the PGFE scheme without the regularization method would fail to accurately capture solution profiles even if thousands of elements are used, which is not the case for the scheme with the regularization method even with hundreds of elements. Impacts of incorporating an adaptive re-meshing method, which is the moving mesh partial differential equation method, into the PGFE scheme are also assessed, demonstrating that it can enhance robustness of the scheme with regularization.

Key Words : Petrov-Galerkin finite element scheme, Hamilton-Jacobi-Bellman equation, regularization method, consistency error analysis, adaptive re-meshing method

\section{INTRODUCTION}

Optimization problems of stochastic dynamical systems, such as extracting surface and ground water resources ${ }^{1}$, managing water quality indices in water bodies $^{2)}$, and assessing movements of robots and organisms in fluids ${ }^{3), 4)}$, can be formulated with the mathematical concept of stochastic control ${ }^{5)}$. Solving a stochastic control problem ultimately reduces to finding a solution to a Hamilton-Jacobi-Bellman equation $(\mathrm{HJBE})^{5)}$, which is a nonlinear and nonconservative parabolic or elliptic differential equation. Analytical solutions to HJBEs are available only for limited number of cases ${ }^{5}$. Their solutions have therefore been numerically approximated in applications. As the HJBEs are nonconservative differential equations, finite element ${ }^{1), 6}$, finite difference $^{7), 8)}$, and semi-Lagrangian schemes ${ }^{9)}{ }^{10)}$ have been used for their numerical resolution. Solutions to some HJBEs encountered in scientific and engineering applications comply with maximum principles $^{11), 12)}$. Their numerical counterparts should possess analogous properties, such as the discrete maximum principles ${ }^{13)}$, ${ }^{14)}$. It is therefore desirable to use an unconditionally stable numerical method for ensuring reliability of the numerical solutions. 
Some researchers found that an appropriate regularization method is necessary for accurately computing numerical solutions to nonlinear PDEs having regularity deficits, such as the advection equations ${ }^{15}$, degenerate diffusion equations ${ }^{16),}{ }^{17)}$, turbulence models ${ }^{18),}{ }^{19)}$, and non-conservative parabolic differential equations associated with stochastic process models ${ }^{20), 21)}$. The authors applied a regularization method to the 1-D HJBE governing optimal migration strategy of individual fishes ${ }^{4), 22)}$. Their computational results implied that numerical solutions to the HJBEs could be reasonably computed with the help of a regularization method even using relatively coarse computational meshes ${ }^{22)}$; however, their rigorous mathematical analysis and detailed assessment of computational performance have not been performed so far, which is a main motivation of this paper.

Solutions to nonlinear differential equations often involve sharp transitions that unconditionally stable numerical schemes cannot always capture because they do not in general perform high computational accuracy ${ }^{23), 24)}$. Numerically approximating solutions to HJBEs would encounter similar computational issues ${ }^{4), 22)}$. Such issues can be overcome with adaptive re-meshing techniques that employ fine numerical resolution where solutions have sharp transitions ${ }^{25)}{ }^{26)}$. One of the adaptive re-meshing techniques that have successfully been used for nonlinear PDEs is the moving mesh partial differential equation (MMPDE) method ${ }^{27}$. Application range of the method is quite wide, ranging from 1-D single $\mathrm{PDEs}^{28), 29)}$ to multi-dimensional system of PDEs ${ }^{30)}$, 31). The MMPDE method solves a parabolic PDE controlling mesh resolution in addition to the equation to be solved ${ }^{32), 33)}$, and does not modify the mesh topology, such as linkage of nodes and elements.

The main purpose of this paper is to examine a regularization method and an MMPDE method for numerically approximating solutions to 1-D HJBEs, focusing in particular on that governing a dynamic energy-minimizing migration strategy of individual fishes ${ }^{4)}$ as a model problem. The HJBEs in this paper have linear diffusion and nonlinear drift terms. Consistency error between true and regularized solutions to the 1-D HJBEs is estimated both theoretically and numerically; the latter is performed with a Petrov-Galerkin finite element (PGFE) ${ }^{34),}{ }^{35}$ ). Numerical analysis on the PGFE scheme with the regularization method and the MMPDE method is performed for assessing their computational performance. Their applicability to engineering problems is also discussed.
The remainder of this paper is organized as follows. Section 2 presents the 1-D HJBE. Section 3 proposes the regularization method and performs its mathematical analysis. Section 4 briefly explains the PGFE scheme. Section 5 performs numerical analysis on the scheme. Section 6 concludes this paper. Appendix contains supplement information not presented in the main text.

In this paper, $C$ represents generic positive constants that do not depend on the regularization parameter $K$, which is rigorously defined later. The norm of a function $g$ in the functional space $H$ is denoted by $\|g\|_{H}$.

\section{MATHEMATICAL MODEL}

\section{(1) Hamilton-Jacobi-Bellman equation}

An HJBE governs a value function subject to a controlled dynamical system represented by a stochastic differential equation (SDE) ${ }^{5}$. An HJBE is a nonconservative parabolic or elliptic differential equation whose spatial dimension equals the degree of freedom of the system. For a stochastic control problem of an autonomous system with a time-independent value function, the HJBE is an elliptic differential equation. For a system with one degree of freedom, the HJBE reduces to an ordinary differential equation subject to boundary conditions. The domain of an HJBE is identified with the range of dependent variables of the system: a 1-D interval for the one-degree case.

This paper considers the time-independent HJBE

$$
D \frac{\mathrm{d}^{2} v}{\mathrm{~d} x^{2}}+b\left(\frac{\mathrm{d} v}{\mathrm{~d} x}\right) \frac{\mathrm{d} v}{\mathrm{~d} x}=f
$$

in the 1-D interval $\Omega=(0, L)$ with $L(>0)$ subject to a Dirichlet boundary condition where $x$ is the 1-D coordinate, $v=v(x)$ is the solution, $D=\operatorname{const}(>0)$ is the diffusion coefficient, $b=b(q)$ with $q \in \mathbb{R}$ is the drift coefficient, and $f=f(x)$ represents source terms independent of the solution $v$. Eq.(1) represents a class of simplest 1-D HJBE encountered in scientific and engineering applications. The Eikonal equations $^{36 \text {, }}$ 37) governing weighted shortest path from the domain boundary are such examples. The HJBE governing a dynamic energy-minimizing migration strategy of individual fishes in open channels is another such example ${ }^{4), 38)}$.

Hereafter, the drift coefficient $b=b(q)$ is set as 


$$
b(q)=V+\frac{1}{2} q \text { for } q \in \mathbb{R}
$$

with a bounded and sufficiently smooth $V=V(x)$ unless otherwise specified. The drift coefficient $b$ in Eq.(2) appears in the 1-D HJBE governing the dynamic energy-minimizing migration strategy of individual fishes in open channels ${ }^{4), 38)}$. The drift coefficient $b(q)$ in Eq.(2) is linear and unbounded in $q$.

\section{(2) Computational issue}

A computational issue encountered in solving Eq.(1) is demonstrated for the constants $V(>0)$ and $f=0$ with the boundary conditions $v(0)=P(>0)$ and $v(L)=0$, which is the simplest case in applications ${ }^{4)}$. The HJBE in this case governs an energy-minimizing migration velocity $u=u(x)$ of individual fishes at each point $x$ in the 1-D open channel $\Omega=(0, L)$ with the uniform flow velocity $V$. The constant $P$ serves as the profit of the fishes gained at the upstream-end $x=0$ where a habitat or a spawning site of fishes exists. The migration velocity $u=u(x)$ is theoretically given in terms of $v$ as ${ }^{4)}$

$$
u=-\frac{\mathrm{d} v}{\mathrm{~d} x},
$$

which leads to the optimal ground velocity of fishes

$$
V_{\mathrm{g}}=V-u=V+\frac{\mathrm{d} v}{\mathrm{~d} x} .
$$

Eq.(4) leads to that the ground velocity $V_{\mathrm{g}}$ is toward the upstream-end $x=0\left(V_{\mathrm{g}}<0\right)$ over $\Omega$ when

$$
0<V<-\frac{\mathrm{d} v}{\mathrm{~d} x},
$$

which is true $\mathrm{if}^{4)}$

$$
Q=P / V L>2 .
$$

A numerical method applied to the HJBE(1) under more realistic conditions should be able to accurately solve this model problem since it is the simplest case. In practice, the Péclet number $\mathrm{Pe}=V L / D$ would be significantly larger than 1 as in the cases of advection-dispersion phenomena of solute. The condition $\mathrm{Pe}=10$ is assumed, which may be small for assuming this consideration but is sufficiently large for demonstrating the computational difficulty.

A classical analytical solution to this problem is available ${ }^{4)}$ and are plotted in Fig. 1. The PGFE scheme ${ }^{34), 35)}$, which is briefly explained in section 4 , is applied to solving Eq.(1) with the initial guess $v=0$. Figs. 2 and 3 compare the numerical and analytical solutions where the domain $\Omega$ is uniformly

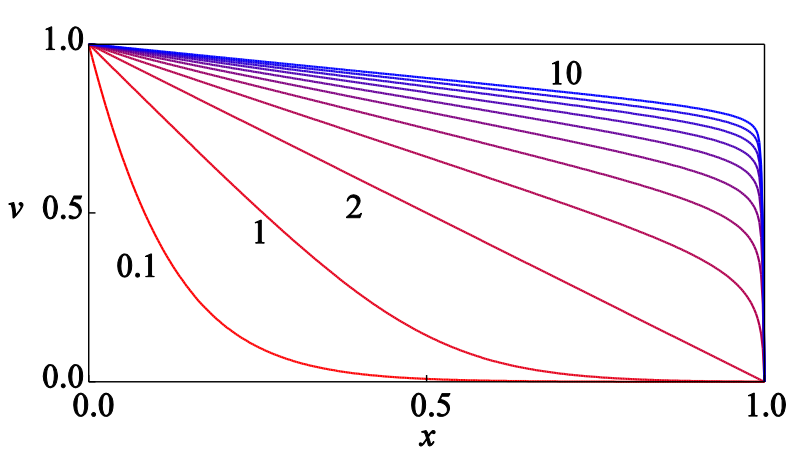

Fig.1 Analytical solutions to Eq.(1). The numbers in the plot area of the figure represents the value of $Q$.

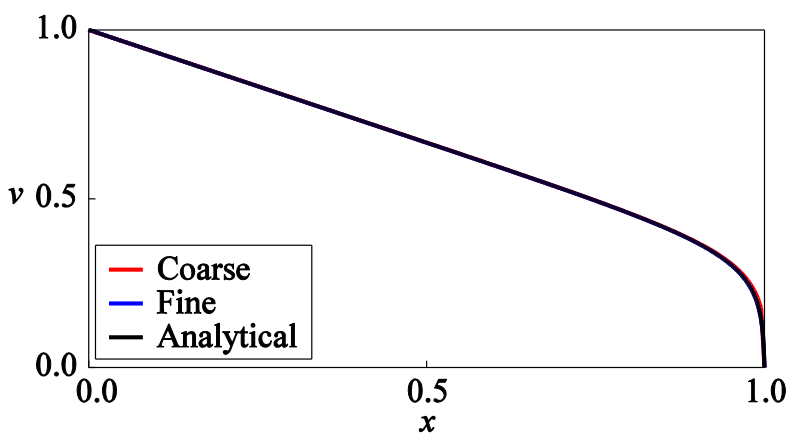

Fig.2 Comparison of numerical and analytical solutions ( $Q=3$ ).

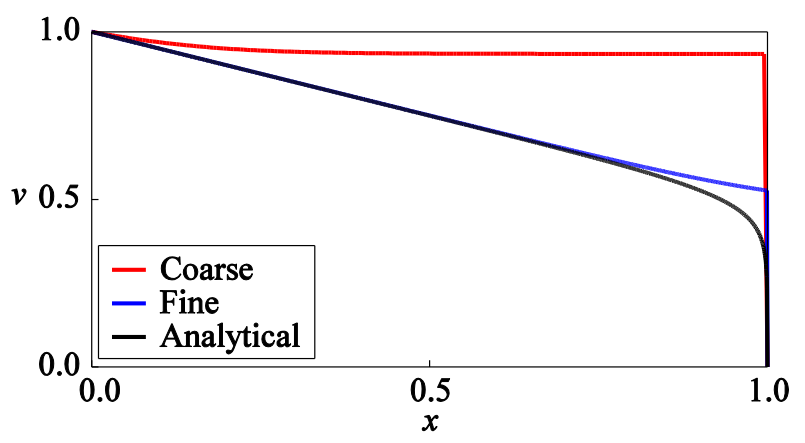

Fig.3 Comparison of numerical and analytical solutions $(Q=4)$.

discretized into fine (2,000 elements) or coarse mesh (200 elements). The domain $\Omega$ and the solution $v$ are normalized with the length $L$ and the profit $P$, respectively. Fig. 2 for $Q=3$ shows reasonable agreement between the numerical and analytical solutions. On the other hand, Fig. 3 for $Q=4$ shows that the numerical solutions significantly deviate from the analytical solution, although they rigorously satisfy the discrete maximum principle. The numerical solution with the coarse mesh has a spurious flat profile except for near the boundary $x=0$. According to Eq.(5), this phenomenon means absence of the upstream migration of fishes $\left(V_{\mathrm{g}}>0\right)$, which is inconsistent with the analytical solution ${ }^{4}$. Empirically, this phenomenon occurs for $Q>3.5$ and becomes 
more significant as $\mathrm{Pe}$ or $Q$ increases. Conventional numerical schemes, such as the first-order fully upwind FD schemes, suffer from the same issue. This phenomenon arises for smaller value of $Q$ with coarser computational mesh. The conventional central schemes fail due to highly advective nature of the solution, which is prominent near $x=L$ in particular Similar or more serious computational issues would be encountered for problems that are more complicated.

\section{(3) Possible causes of computational inaccuracy}

One possible cause of the above-presented issue is nonlinearity and unboundedness of the drift coefficient $b$, which cause the sharp transition of the solution $v$ near the boundary $x=L$. In addition, the nonlinearity and unboundedness of the drift coefficient $b$ does not always guarantee well-posedness of the problem because of regularity deficits ${ }^{39)}$. The available classical analytical solution ${ }^{4)}$ is an exceptional case. We conjectured that application of an appropriate regularization method to the drift coefficient $b$ that artificially bounds it may overcome this issue. Although this is a heuristic consideration, section 4 demonstrates that regularizing the drift coefficient $b$ significantly improves computational performance of the PGFE scheme.

\section{REGULARIZATION METHOD}

The regularization method for the drift coefficient $b$ of the HJBE(1) is presented. Mathematical analysis on the method is also performed for theoretically estimating consistency errors between solutions to the original and regularized HJBEs.

\section{(1) Regularization kernel}

The regularization kernel, which is applied to the derivative $\frac{\mathrm{d} v}{\mathrm{~d} x}$ in the drift coefficient $b$ of the $\operatorname{HJBE}(1)$, is proposed as

$$
h_{K, a}(q)=K q\left(K^{2 a}+q^{2 a}\right)^{\frac{-1}{2 a}}
$$

for $q \in \mathbb{R}$ where the real number $K(>0)$ and the bounded natural number $a$ are regularization parameters. By Eq.(7), the function $h_{K, a}(q)$ is increasing in $q$ for fixed $K$ and $a$. The function $h_{K, a}(q)$ is bounded as

$$
\left|h_{K, a}(q)\right| \leq K
$$

for all $q \in \mathbb{R}$. Eq.(7) leads to

$$
\frac{\partial h_{K, a}(q)}{\partial q}=K^{2 a+1}\left(K^{2 a}+q^{2 a}\right)^{-\left(\frac{1}{2 a}+1\right)},
$$

which further leads to the inequalities

$$
\left|\frac{\partial h_{K, a}(q)}{\partial q}\right| \leq C \text { and }\left|q \frac{\partial h_{K, a}(q)}{\partial q}\right| \leq C K
$$

for bounded $q$. The absolute difference between $q$ and $h_{K, a}(q)$ is estimated as

$$
\left|q-h_{K, a}(q)\right|=|q|\left(1-K\left(K^{2 a}+q^{2 a}\right)^{\frac{-1}{2 a}}\right) \leq C K^{-2}
$$

for bounded $q$. This is because defining the univariate function $g=g(K)$ as

$$
(0 \leq) g(K)=1-K\left(K^{2 a}+q^{2 a}\right)^{\frac{-1}{2 a}}
$$

yields the estimate

$$
\left|\frac{\partial}{\partial K} g(K)\right| \leq K^{-(1+2 a)} q^{2 a} .
$$

Eq.(13) leads to the inequality

$$
\begin{aligned}
\left|q-h_{K, a}(q)\right| & =q|g(K)| \\
& =q\left|\int_{+\infty}^{K} \frac{\partial}{\partial K} g\left(K_{1}\right) \mathrm{d} K_{1}\right| \\
& \leq q^{2 a+1}\left|\int_{+\infty}^{K}\left(K_{1}\right)^{-(1+2 a)} \mathrm{d} K_{1}\right| \\
& \leq \frac{1}{2 a} q^{2 a+1} K^{-2}
\end{aligned}
$$

because $\lim _{K \rightarrow+0} g(K)=0$. The convergence rate of the difference $\left|q-h_{K, a}(q)\right|$ is therefore at least second-order in $K^{-1}$.

\section{(2) Regularized HJBE}

The regularized HJBE is defined with the regularization kernel $h_{K, a}(q)$ in Eq.(7) as

$$
D \frac{\mathrm{d}^{2} v_{K, a}}{\mathrm{~d} x^{2}}+b\left(h_{K, a}\left(\frac{\mathrm{d} v_{K, a}}{\mathrm{~d} x}\right)\right) \frac{\mathrm{d} v_{K, a}}{\mathrm{~d} x}=f
$$

where $v_{K, a}$ is its solution. Eq.(15) is subject to the same boundary conditions with the original HJBE in Eq.(1). The drift coefficient of Eq.(15), which is denoted by $b_{K, a}$, is given by

$$
b_{K, a}\left(\frac{\mathrm{d} v}{\mathrm{~d} x}\right)=b\left(h_{K, a}\left(\frac{\mathrm{d} v}{\mathrm{~d} x}\right)\right) .
$$

Eq.(16) is expressed with Eq.(2) as 


$$
b_{K, a}(q)=V+\frac{1}{2} h_{K, a}(q) .
$$

The only difference between the original and the regularized HJBEs is their drift coefficients. The drift coefficient $b_{K, a}$, unlike the original counterpart $b$, is bounded for fixed $K$ and $a$. This is because

$$
\frac{\partial b_{K, a}(q)}{\partial q}=V+\frac{1}{2} \frac{\partial h_{K, a}(q)}{\partial q} \leq C(1+K)<+\infty
$$

for bounded $K$. In addition, the drift coefficients $b$ and $b_{K, a}$ satisfy the inequality

$$
0<\left|b_{K, a}(q)-b(q)\right| \leq C K^{-2}
$$

for bounded $q \in \mathbb{R}$. This is proven as follows. For a $q \geq 0$, a direct calculation yields

$$
\begin{aligned}
& 2\left(b(q)-b_{K, a}(q)\right) \\
= & q\left(1+\left(q K^{-1}\right)^{2 a}\right)^{\frac{-1}{2 a}}\left(\left(1+\left(q K^{-1}\right)^{2 a}\right)^{\frac{1}{2 a}}-1\right) . \\
\leq & q\left(\left(1+\left(q K^{-1}\right)^{2 a}\right)^{\frac{1}{2 a}}-1\right)
\end{aligned}
$$

Substituting the analytical estimate

$$
\left(1+\left(q K^{-1}\right)^{2 a}\right)^{\frac{1}{2 a}}-1 \leq C K^{-2}
$$

to Eq.(20) leads to Eq.(19) because the function $1+\left(q K^{-1}\right)^{2 a}$ is odd in $K^{-1}$ for bounded. The proof for $q<0$ is straightforwardly obtained by replacing $q$ with $-q$ in the above-presented proof.

By the Schauder's fixed point theorem ${ }^{39)}$, the regularized $\operatorname{HJBE}(15)$ has a unique classical solution. Naively, it is expected to approach the solution to the original $\operatorname{HJBE}(1)$ as the value of $K$ increases (equivalently, as regularization becomes weak). Role of the parameter $a$ is mathematically analyzed in the following sub-sections. The next sub-section gives theoretical estimates of consistency errors between solutions to the original and regularized HJBEs, which demonstrate consistency of the present regularization method.

\section{(3) Consistency error analysis}

The abbreviations

$$
q(x)=\frac{\mathrm{d} v}{\mathrm{~d} x}, q_{K, a}(x)=\frac{\mathrm{d} v_{K, a}}{\mathrm{~d} x}, \text { and } \eta_{K, a}=q-q_{K, a}
$$

are used in this subsection for the sake of brevity. The HJBEs(1) and (15) are assumed to have unique classical solutions, which is true for sufficiently smooth and bounded $V$ and $f$. The inequality

$$
|v|,|q|,\left|v_{K, a}\right|,\left|q_{K, a}\right|<C
$$

is thus assumed to be true. The equality

$$
\begin{aligned}
D \frac{\mathrm{d}}{\mathrm{d} x} \eta_{K, a} & =-\left(b(q)-b_{K, a}\left(q_{K, a}\right)\right) \\
& =-V \eta_{K, a}-\frac{1}{2}\left(q^{2}-q_{K, a} h_{K, a}\left(q_{K, a}\right)\right)
\end{aligned}
$$

directly follows from Eqs.(1) and (15). By Eq.(19), the inequality

$$
0<h_{K, a}\left(q_{K, a}\right)-q_{K, a} \leq C K^{-2}
$$

follows with $q_{K, a}<0, \quad h_{K, a}\left(q_{K, a}\right)<0 \quad, \quad$ and $-h_{K, a}\left(q_{K, a}\right)<-q_{K, a}$ in the domain $\Omega$. The first condition follows from an elliptic maximum principle of the regularized HJBE, namely its solution in $\Omega$ is monotonically decreasing in $x$. By Eqs.(23) and (25), the inequality

$$
0<-q_{K, a} h_{K, a}\left(q_{K, a}\right)+\left(q_{K, a}\right)^{2} \leq C K^{-2}
$$

is deduced. Note that the equality

$$
\begin{aligned}
& -\frac{1}{2}\left(q^{2}-q_{K, a} h_{K, a}\left(q_{K, a}\right)\right) \\
= & -\frac{1}{2}\left(q+q_{K, a}\right) \eta_{K, a}-\frac{1}{2} q_{K, a}\left(q_{K, a}-h_{K, a}\left(q_{K, a}\right)\right)
\end{aligned}
$$

holds. Eq.(26) with Eq.(27) leads to

$$
\frac{\mathrm{d}}{\mathrm{d} x}\left(e^{F} \eta_{K, a}\right)=-\frac{1}{2} e^{F} q_{K, a}\left(q_{K, a}-h_{K, a}\left(q_{K, a}\right)\right)
$$

with the univariate function $F=F(x)$ given by

$$
\begin{aligned}
F & =\frac{1}{D} \int_{0}^{x}\left(V+\frac{q+q_{K, a}}{2}\right) \mathrm{d} y \\
& =\frac{1}{D}\left(\int_{0}^{x} V \mathrm{~d} y-P+\frac{v+v_{K, a}}{2}\right)
\end{aligned}
$$

The function $F$ is bounded in $x$ because of an elliptic maximum principle of the original and regularized HJBEs, which gives the inequality

$$
0 \leq v, v_{K, a} \leq P<+\infty .
$$

The exponentials $e^{ \pm F}$ are bounded as well. Integrating both hand-sides of Eq.(28) by $x$ leads to

$$
\begin{aligned}
& \eta_{K, a}(x)-e^{-F} \eta_{K, a}(0) \\
& =-\frac{1}{2} e^{-F} \int_{0}^{x} e^{F} q_{K, a}\left(q_{K, a}-h_{K, a}\left(q_{K, a}\right)\right) \mathrm{d} y
\end{aligned}
$$

where $y$ is the variable of integration. Integrating 
both hand-sides of Eq.(31) by $x$ yields

$$
\begin{aligned}
& v(x)-v_{K, a}(x)-\eta_{K, a}(0) \int_{0}^{x} e^{-F} \mathrm{~d} y \\
& =-\frac{1}{2} \int_{0}^{x} e^{-F} \int_{0}^{y} e^{F} q_{K, a}\left(q_{K, a}-h_{K, a}\left(q_{K, a}\right)\right) \mathrm{d} y \mathrm{dz}
\end{aligned}
$$

where $z$ is the variable of integration. Taking absolute values of the both hand-sides of Eq.(32) yields

$$
\begin{aligned}
& \left|v(x)-v_{K, a}(x)-\eta_{K, a}(0) \int_{0}^{x} e^{-F} \mathrm{~d} y\right| \\
= & \left|\frac{1}{2} \int_{0}^{x} e^{-F} \int_{0}^{y} e^{F} q_{K, a}\left(q_{K, a}-h_{K, a}\left(q_{K, a}\right)\right) \mathrm{d} y \mathrm{dz}\right| \\
\leq & \frac{1}{2} L^{2}\left\|e^{-F}\right\|_{L^{\infty}}\left\|e^{F}\right\|_{L^{\infty}}\left\|q_{K, a}\right\|_{L^{\infty}}\left\|q_{K, a}-h_{K, a}\left(q_{K, a}\right)\right\|_{L^{\infty}} \\
\leq & C K^{-2}
\end{aligned}
$$

by Eq.(26) and the boundedness of $e^{ \pm F}$. Taking $x=L$ in Eq.(33) yields

$$
\left|\eta_{K, a}(0)\right| \leq C K^{-2}
$$

since $v(L)=v_{K, a}(L)$. Eq.(32) is rewritten as

$$
\begin{aligned}
& v(x)-v_{K, a}(x) \\
& =\eta_{K, a}(0) \int_{0}^{x} e^{-F} \mathrm{~d} y \\
& -\frac{1}{2} \int_{0}^{x} e^{-F} \int_{0}^{y} e^{F} q_{K, a}\left(q_{K, a}-h_{K, a}\left(q_{K, a}\right)\right) \mathrm{d} y \mathrm{dz}
\end{aligned}
$$

Application of Eqs.(26), (34), and the boundedness of $e^{ \pm F}$ to Eq.(35) yields

$$
\left|v(x)-v_{K, a}(x)\right| \leq C K^{-2}
$$

at each $x \in \Omega$, leading to the consistency error

$$
\left\|v-v_{K, a}\right\|_{L^{\infty}} \leq C K^{-2} .
$$

Eq.(37) means that Eq.(15) is consistent with Eq.(1) for $K \rightarrow+\infty$ and shows that the convergence rate is at least $O\left(K^{-2}\right)$. In addition, Eq.(37) leads to

$$
\left\|v-v_{K, a}\right\|_{L^{p}} \leq C K^{-2}
$$

for $1 \leq p<+\infty$.

\section{(4) On optimal numerical regularization}

The regularization method has two parameters $K$ and $a$ to be specified a priori. The parameter $a$ does not affect the convergence rate $K^{-2}$, showing its relative insensitivity to the convergence results. The value $a=2$ is thus assumed in what follows for the sake of simplicity. The parameter $K$ should be chosen sufficiently large so that the consistency error between the solution to Eq.(1) and that to Eq.(15) is sufficiently small, but should also be sufficiently small so that accurate numerical solutions suppressing the spurious profiles are obtained.

In numerical computation, it is reasonable to consider the parameter $K$ as a decreasing function of the element size $\Delta x$, so that the regularization effect becomes negligible when sufficiently fine computational meshes are used. The consistency error estimates derived in the previous section provides a guideline to choose the parameter $K$ as a function of the element size $\Delta x$. Consider a numerical method whose computational accuracy is $O\left((\Delta x)^{k}\right)$ with some $k(>0)$ in the usual $L^{p}$ sense. By Eq.(38), the computational error $e$ in the $L^{p}$ sense between the true solution $v$ and the numerical approximation $v_{K, a, \Delta x}$ of the regularized solution can be roughly estimated as

$$
\begin{aligned}
e & =\left\|v-v_{K, a, \Delta x}\right\|_{L^{p}} \\
& \leq\left\|v-v_{K, a}\right\|_{L^{p}}+\left\|v_{K, a}-v_{K, a, \Delta x}\right\|_{L^{p}} . \\
& =O\left(K^{-2}\right)+O\left((\Delta x)^{k}\right)
\end{aligned}
$$

Assume that the parameter $K$ is effectively scaled as $K=C(\Delta x)^{-\alpha}$ with a constant $\alpha(>0)$. Eq.(39) in this case reduces to

$$
e \leq O\left((\Delta x)^{2 \alpha}\right)+O\left((\Delta x)^{k}\right)=O\left((\Delta x)^{\min (2 \alpha, k)}\right) .
$$

By Eq.(40), numerical solutions using the regularization method converge to the true, non-regularized solutions without degrading the computational accuracy if $\alpha \geq \frac{1}{2} k$. Since most of the numerical methods at least perform the accuracy $O(\Delta x)$, the power $\alpha$ should be chosen as

$$
\alpha \geq \frac{1}{2} \text {. }
$$

Eq.(41) is numerically verified in the next section.

\section{NUMERICAL METHOD}

\section{(1) Petrov-Galerkin finite element scheme}

The PGFE scheme used in this paper is a conforming FE scheme based on the fitting technique, in which both the trial and test functions are determined from analytical solutions to local two-point boundary value problems. Spatial and temporal discretization procedures of the PGFE scheme are presented in the literatures ${ }^{34), 35)}$, and are therefore not presented here.

For time-independent linear problems, numerical solutions with the PGFE scheme guarantee the el- 
liptic discrete maximum principle. In addition, the numerical solutions satisfy the uniform convergence toward the corresponding exact solutions with respect to the diffusion coefficient $D$ if the other known functions are sufficiently regular, which cannot be achieved in most of the conventional nu-

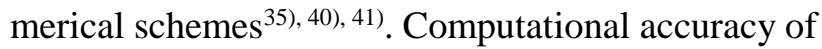
the PGFE scheme for linear problems has been verified in detail, and theoretical and numerical analyses indicated that its computational accuracy in space is at least first order in the $L^{\infty}$-sense at each node. In this paper, each numerical solution is computed with a pseudo temporal integration method inspired from an artificial compressibility method in the continuity equation of incompressible hydrodynamic models ${ }^{42}$.

In addition to the above-mentioned PGFE scheme, there exist a number of $\mathrm{FE}$ schemes of the Petrov-Galerkin type; such numerical schemes can be broadly categorized into those with high-order computational accuracy while not always spatially monotone ${ }^{43), 44)}$, those with low-order computational accuracy due to the fully upwind nature subject to larger numerical diffusion coefficient ${ }^{40)}$, or those numerically equivalent with the PGFE scheme ${ }^{45)}$, 46). The present HJBE is nonlinear and its solution derivative determines both magnitude and direction of the optimal swimming velocity of individual fishes, which is an ecologically important quantity. Its numerical computation thus requires accurate resolution of both the solution and its derivative, which is also true for generic HJBEs in other applications. The non-monotonic schemes, which would inaccurately compute the derivative, should therefore not be used for solving HJBEs. It has been checked that the present and lower accurate scheme give not significantly different computational results for the present HJBE because of the strong advection effect. This paper therefore focuses solely on numerical analysis on the PGFE scheme.

\section{(2) Adaptive re-meshing method}

An adaptive re-meshing method, which is referred to as the MMPDE method, is introduced in this paper. Details of the method are presented in the literatures $^{27,}, 43$, and are therefore briefly explained in this paper. The MMPDE method used in this paper is their time-independent counterpart. In the present MMPDE method, each computational node is adaptively moved according to the monitor function $M$, which serves as a measure of computational errors. Using appropriate monitor functions can control length of each element so that sharp transitions of solution profiles are effectively resolved.
The governing equation of the nodal position $x=x(\xi)$ as a function $\xi$ in another 1-D interval $U=(0,1)$ is determined by solving the nonlinear diffusion equation

$$
\frac{\mathrm{d}}{\mathrm{d} \xi}\left(M \frac{\mathrm{d} x}{\mathrm{~d} \xi}\right)=0 .
$$

subject to the monitor function ${ }^{43)}$

$$
M(x)=\sqrt{\lambda\left(\frac{\mathrm{d} v}{\mathrm{~d} x}\right)^{2}+\delta} \text { with } \lambda=\left(\sup _{x \in \Omega}\left|\frac{\mathrm{d} v}{\mathrm{~d} x}\right|+\varepsilon\right)^{-2}
$$

where $\delta$ and $\varepsilon$ are positive constants, both of which are set as $10^{-7}$ in this paper; the former has been tuned so that numerical stability is maintained throughout computation and the latter is used simply for avoiding division by zero.

The derivative $\frac{\mathrm{d} v}{\mathrm{~d} x}$ of $v$ appearing in Eq.(43) is evaluated in each element. In each computation with the MMPDE method, an HJBE and Eq.(42) are simultaneously discretized for obtaining converged numerical steady solutions of $v$ and $x$. Using smaller $\delta$ leads to smaller elements at the areas where the solution $v$ has sharp transitions; however, degradation of computational efficiency in the iterative computation step would occur due to highly varying monitor functions $M$ in space. This issue is not focused on in this paper, but will be investigated in future researches. For the computational cases presented in the later section, using the MMPDE almost doubles computation time.

\section{APPLICATIONS}

The PGFE scheme is applied to the 1-D HJBE governing the migration of individual fishes focused on in section 2. The regularization parameter $K$ is assumed to be normalized by the constant $V$. This sub-section assumes $Q=P / V L>3$ that cover the cases with $Q>3.5$ where spurious numerical solutions have been obtained.

\section{(1) Optimal value of the parameter $K=K_{\mathrm{opt}, \Delta x}$}

Firstly, the optimal value of the regularization parameter $K=K_{\mathrm{opt}, \Delta x}$, which minimizes the nodal $L^{2}$-error $e_{2}$ between true and regularized numerical solutions, is identified with a sensitivity equation-based technique ${ }^{44)}$ explained in Appendix. 
The domain $\Omega$ is uniformly divided into a computational mesh with 200, 400, or 800 elements. Figs. 4 and 5 plot computed $K=K_{\text {opt }}$ for the Péclet numbers $\mathrm{Pe}=10$ and $\mathrm{Pe}=20$, respectively. The computational results presented in the figures show monotonic increase of $K_{\text {opt }}$ with respect to the total number of elements, the normalized boundary value $Q$, and the inverse of the Péclet number $\mathrm{Pe}^{-1}$. The computational results suggest the relationship

$$
K_{\mathrm{opt}, \Delta x} \approx 2 K_{\mathrm{opt}, 2 \Delta x}
$$

for $Q>3.5$ and each Pe, which means

$$
K_{\mathrm{opt}, \Delta x}=O\left((\Delta x)^{-\alpha}\right)
$$

with $\alpha=1$. Eq.(45) is consistent with Eq.(41) for $k=1$ and the fact that accuracy of the PGFE scheme is at least first-order in space. Eq.(44) is not well satisfied for $Q<3.5$ where numerical solutions without spurious profiles are obtained. This result implies that the heuristically derived Eq.(41) would not hold for the small $Q$.

Figs. 6 and 7 plot the $L^{2}$-errors $e_{2}$ between the regularized numerical solutions with the parameter $K=K_{\text {opt }}$ and analytical solutions for $\mathrm{Pe}=10$ and $\mathrm{Pe}=20$, respectively. The errors $e_{2}$ in the figures are at most $O\left(10^{-5}\right)$ for hundreds of elements. Estimated convergence rates of the numerical solutions from the computational results are almost 1. In Fig. 6 , the errors $e_{2}$ with 400 elements are larger than those with 200 elements for $Q<3.5$. This phenomenon implies that regularizing the drift coefficient does not always effectively work for relatively small $Q$, which was also implied from the results presented in Figs. 4 and 5 as pointed out at the end of the previous paragraph. However, the errors $e_{2}$ in these cases are still at the order of $O\left(10^{-5}\right)$, which are considered to be sufficiently small values.

\section{(2) Impacts of using the MMPDE method}

Fig. 8 compares the regularized numerical solutions and the analytical solution for $K=10+20 i$ with the integers $1 \leq i \leq 9$ where $K_{\mathrm{opt}, \Delta x}=88$. The model parameters are $Q=6$ and $\mathrm{Pe}=10$. The numerical solutions are monotonically increasing in $K$ at each point $x \in \Omega$. Fig. 8 indicates that using too large or too small $K$ would give inaccurate numerical solutions, implying that robustness of the regularization method should be enhanced.

Impacts of incorporating the MMPDE method into

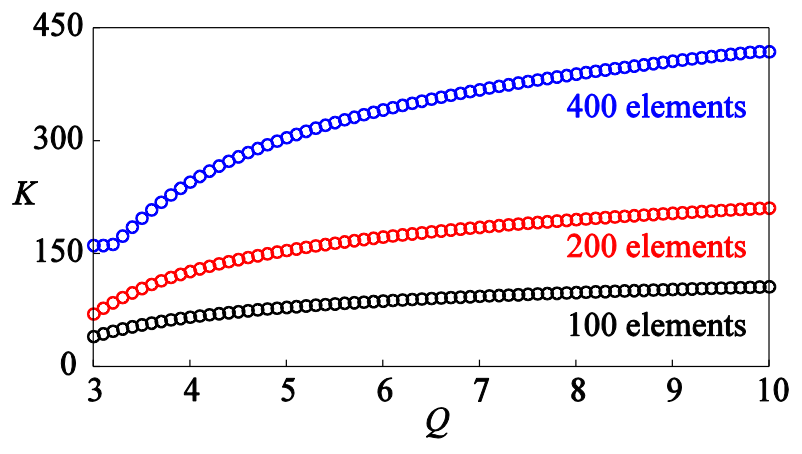

Fig.4 Computed $K_{\text {opt }}$ for $\mathrm{Pe}=10$.

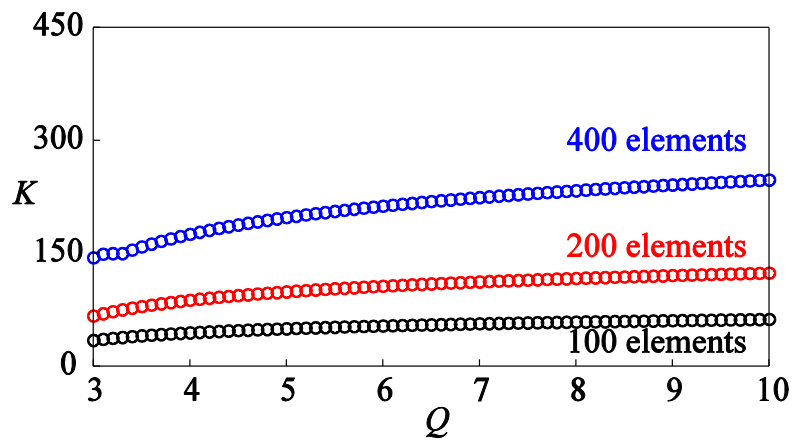

Fig.5 Computed $K_{\text {opt }}$ for $\mathrm{Pe}=20$.

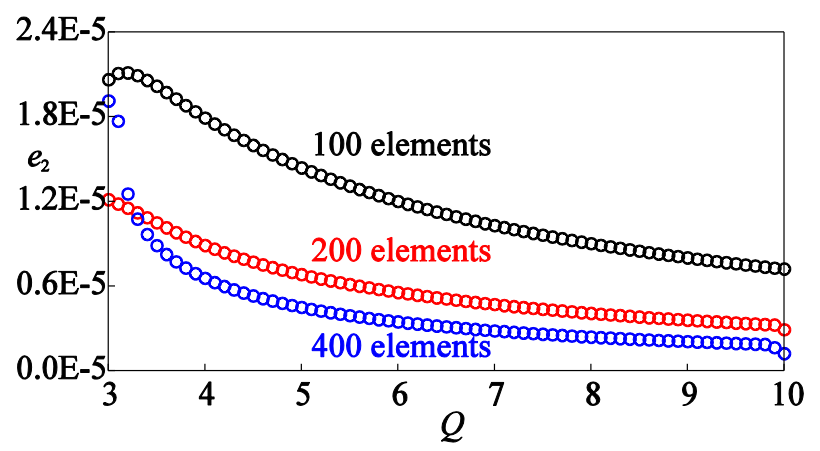

Fig.6 The $L^{2}$-errors $e_{2}$ between the regularized numerical solutions with $K=K_{\mathrm{opt}, \Delta x}$ and analytical solutions for $\mathrm{Pe}=10$.

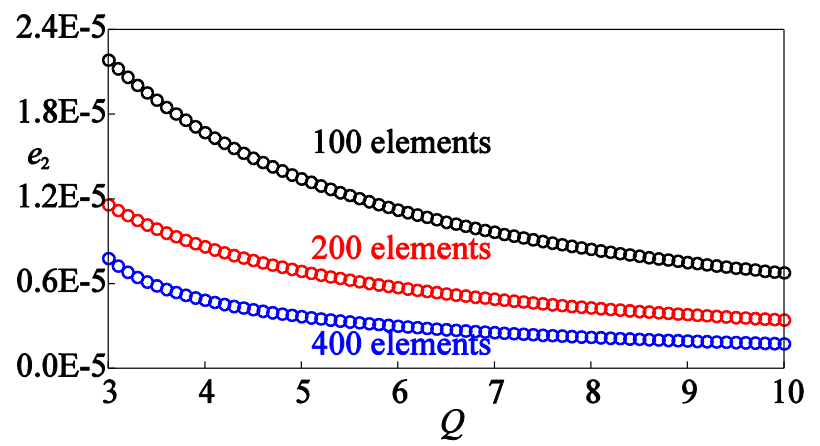

Fig.7 The $L^{2}$-errors $e_{2}$ between the regularized numerical solutions with $K=K_{\mathrm{opt}, \Delta x}$ and analytical solutions for $\mathrm{Pe}=20$.

the PGFE scheme on its robustness are finally assessed. Fig. 9 compares the regularized numerical solution with 100 uniform elements $\left(K=K_{\mathrm{opt}, \Delta x}\right)$, 
non-regularized numerical solution with 2,000 uniform elements $(K=+\infty)$, regularized numerical solution using the MMPDE with 100 uniform elements ( $K=900$ ), and the analytical solution for $Q=6$ and $\mathrm{Pe}=10$.

The computational results in Fig. 9 indicate an advantage of using the MMPDE method in solving the HJBE, allowing larger value of the regularization parameter $K$ (weaker regularization effect) for computing numerical solutions without spurious profiles. Incorporating the MMPDE into the present numerical method thus enhances its robustness, which can potentially improve computational accuracy of regularized numerical solutions with relatively large $K\left(>K_{\text {opt }}\right)$. Similar computational results are obtained for other $Q=O\left(10^{\circ}\right)(>3.5)$. Numerical solutions based on the MMPDE method without the regularization method are not significantly more accurate than those without both of the methods as presented in Fig.10, indicating importance of regularizing the drift coefficient.

\section{(3) Remarks on the numerical method}

Theoretical aspects of the present numerical method have mainly been dealt with in this paper. This sub-section discusses advantages and disadvantages of the numerical method.

A remarkable advantage of using the present regularization method is that it can be easily implemented into a variety of numerical methods for solving the HJBEs in scientific and engineering applications, because it only modifies the drift coefficient. It is potentially applicable to real problems where the known functions, and initial and boundary conditions have lower regularity than those considered in this paper. Realizing the regularization method in a computer program does not encounter technical difficulties.

In some engineering applications, such as analyzing migration of individual fishes in river and canal networks ${ }^{4), 38)}$, 1-D HJBEs have to be numerically solved on connected graphs. A connected graph consists of a number of 1-D reaches glued via 0-D junctions. In such a case, the present PGFE scheme with the regularization method and the MMPDE method can be directly used if appropriate internal boundary conditions are specified at junctions. Implementing the regularization method to the existing multi-dimensional numerical schemes ${ }^{1),}{ }^{45)}$ is also straightforward. However, its performance may depend on the problems to be solved and on numerical methods used in computation. It has been numerically checked that the present regularization method

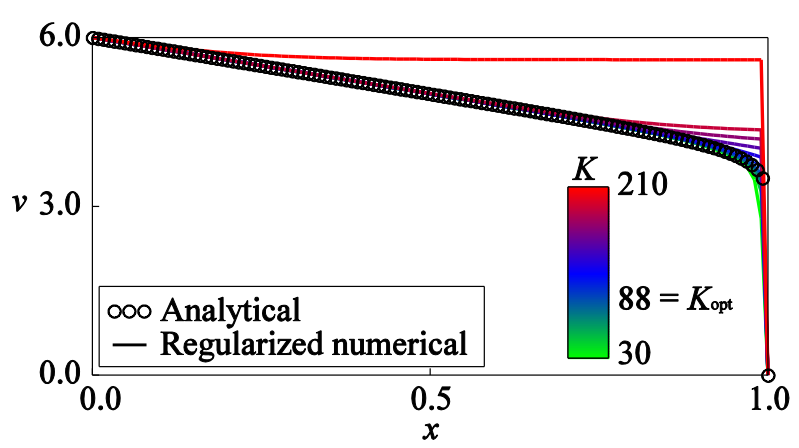

Fig.8 Comparison of the regularized numerical and analytical solutions for $Q=6$ and $\mathrm{Pe}=10$.

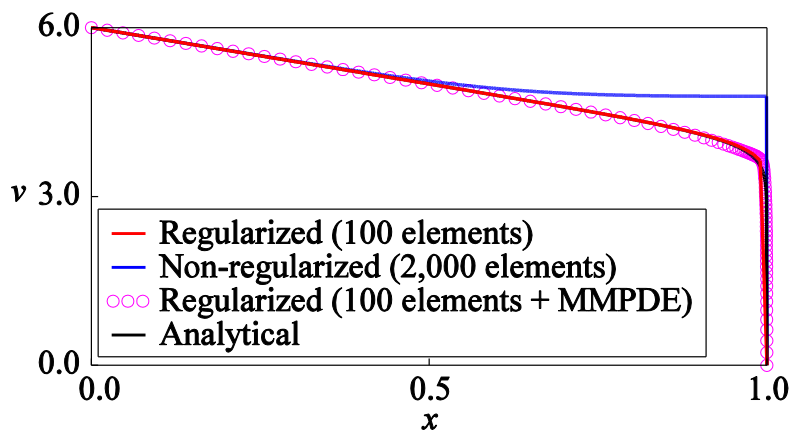

Fig.9 Comparisons of the numerical and analytical solutions for $Q=6$ and $\mathrm{Pe}=10$.

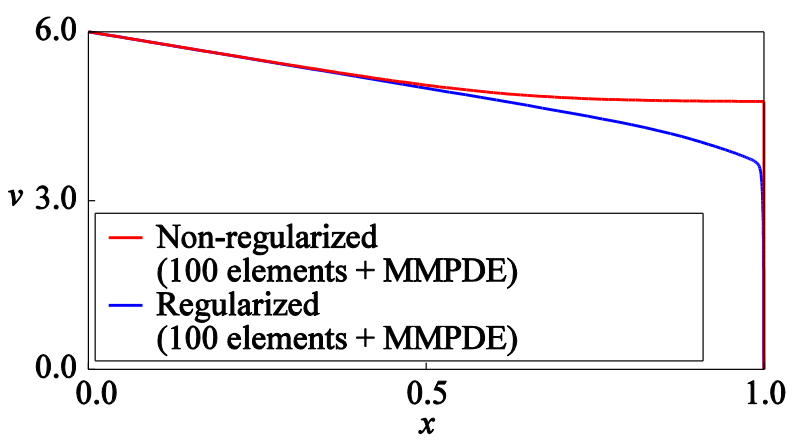

Fig.10 Comparisons of the regularized and non-regularized numerical solutions with the MMPDE for $Q=6$ and $\mathrm{Pe}=10$.

also effectively works for a horizontally 2-D counterpart of the HJBE governing migration of individual fishes around hydraulic structures.

A disadvantage of using the present numerical method is the lack of criteria for determining the parameter $K$ for the problems that are more complicated than that considered in this paper, such as the problems with spatially varying known functions and unsteady problems. A possible extension of the present regularization method to such problems is considering the regularization parameter $K$ as a mesh- and/or solution- dependent parameter. This remains as a research topic to be addressed in future. 


\section{CONCLUSIONS}

A PGFE scheme with a regularization method for numerically solving 1-D time-independent HJBEs was presented. Theoretical consistency errors between the true and regularized solutions were derived, demonstrating mathematical consistency of the present regularization method. The optimal regularization parameter $K=K_{\mathrm{opt}}$ for each fixed computational mesh was identified by minimizing the $L^{2}$-error using a sensitivity equation-based numerical technique. The PGFE scheme with the parameter $K_{\text {opt }}$ successfully computed numerical solutions to the HJBEs with the unbounded drift coefficient. The identified parameter $K_{\text {opt }}$ was inversely proportional to the element size $\Delta x$, which serves as an admissible parameterization according to the results of the theoretical analysis in this paper. The computational results using the MMPDE method indicated that it could enhance robustness of the present numerical method, widening the acceptable range of the regularization parameter $K$ for computing accurate numerical solutions without spurious profiles.

In conclusion, this paper demonstrated that the present numerical method serves as an effective tool for solving the 1-D HJBEs with the unbounded drift coefficient. Future researches will address further mathematical analysis on the regularization method under weaker assumption: namely, consistency error analysis in the context of weak solutions. Other issues to be addressed include assessing impacts of the regularization method on performance of higher-order numerical methods, which would lead to different scaling of the parameter $K_{\text {opt }}$ as the element size $\Delta x$. Applicability and limitation of the present regularization method to the HJBEs with stronger nonlinearity and those under conditions that are more realistic will also be investigated in future researches.

ACKNOWLEDGMENT: The authors thank to Mr. Yusuke Imoto, who is a doctoral student of Graduate School of Science, Kyushu University, Japan, for his helpful comments for improving mathematical presentations of this research. This research was funded by the grant-in-aid for scientific research No. 15H06417 from the Japan Society for the Promotion of Science.

\section{APPENDIX Numerical Optimization Method}

The sensitivity equation-based numerical optimization method for identifying the parameter value $K=K_{\mathrm{opt}, \Delta x}$ is explained. For a fixed computational mesh, the $L^{2}$-error between an analytical solution and the regularized numerical counterpart is

$$
e_{2}=e_{2}(K)=\frac{1}{2} \sum_{i=1}^{N}\left(v_{i}-v_{K, a, \Delta x, i}\right)^{2},
$$

which can be regarded as a function of $K$ where the subscript $i$ specifies the nodes. Assuming that $e_{2}$ is Fréchet differentiable with respect to $K$, By Eq.(46), the Fréchet derivative $\frac{\delta e_{2}}{\delta K}$ is given as

$$
\frac{\delta e_{2}}{\delta K}=-\frac{1}{2} \sum_{i=1}^{N} \frac{\delta v_{K, a, \Delta x, i}}{\delta K}\left(v_{i}-v_{K, a, \Delta x, i}\right) .
$$

The optimal $K$ minimizing the error functional, which is $K_{\mathrm{opt}, \Delta x}$, then satisfies

$$
\begin{aligned}
& \left.\frac{\delta e_{2}}{\delta K}\right|_{K=K_{\text {op }, \Delta x}} \\
& =-\left.\sum_{i=1}^{N} \frac{\delta v_{K, a, \Delta x, i}}{\delta K}\right|_{K=K_{\text {opp }, \Delta x}}\left(v_{i}-\left.v_{K, a, \Delta x, i}\right|_{K=K_{\mathrm{opp}, \Delta x}}\right)=0
\end{aligned}
$$

The governing differential equation of the Fréchet derivative $S=\frac{\delta v_{K, a, \Delta x}}{\delta K}$, which is the sensitivity of $v_{K, a, \Delta x}$ on $K$, is formally derived by differentiating Eq.(1) with respect to $K$ as

$$
D \frac{\mathrm{d}^{2} S}{\mathrm{~d} x^{2}}+\gamma \frac{\mathrm{d} S}{\mathrm{~d} x}+\omega S=0
$$

with the coefficients

$$
\gamma=\frac{\left(2 K^{2 a}+q^{2 a}\right) h_{K, a}(q)}{2\left(K^{2 a}+q^{2 a}\right)}, \omega=\frac{q^{2 a+1} h_{K, a}(q)}{2 K\left(K^{2 a}+q^{2 a}\right)}
$$

for $q=\frac{\mathrm{d} v_{K, a}}{\mathrm{~d} x}$. Eq.(49) is subject to the homogenous

Dirichlet condition on the boundary of $\Omega$ because $K$ does not affect the original boundary conditions. Eq.(49) is a linear differential equation of $S$ and is solved with the PGFE scheme. The value of $K_{\mathrm{opt}, \Delta x}$ is iteratively computed using the following numerical algorithm.

\section{(Numerical optimization algorithm)}

1. Set an initial guess of $K$.

2. Compute nodal $v_{K, a, \Delta x}$ and $S$.

3. Compute $\frac{\delta e_{2}}{\delta K}$ using the updated $v_{K, a, \Delta x}$ and $S$. 
4. Compute the increment of $K$ as $\delta K=\varepsilon_{1} \frac{\delta e_{2}}{\delta K}$ with a constant $\varepsilon_{1}(>0)$.

5. If the condition $|\delta K| \leq \varepsilon_{2}$ is satisfied with a constant $\varepsilon_{2}(>0)$, then output $K_{\mathrm{opt}, \Delta x}=K$ and terminate the algorithm. If $|\delta K| \leq \varepsilon_{2}$ is not satisfied, then go to 6 .

6. Update $K$ as and go to 2 .

The values of and are empirically set as and in this paper. Choosing smaller and does not significantly affect the presented results.

\section{REFERENCES}

1) Unami, K., Mohawaesh, O., Sharifi, E., Takeuchi, J., and Fujihara, M. : Stochastic modelling and control of rainwater harvesting systems for irrigation during dry spells, J. Clean. Prod., Vol. 88, pp. 185-195, 2015

2) Athanassoglou, S. : Dynamic nonpoint-source pollution control policy: Ambient transfers and uncertainty, J. Econ. Dyn. Control, Vol. 34, No. 12, pp. 2494-2509, 2010.

3) Esfahani, P.M., Chatterjee, D., and Lygeros, J. : On a problem of stochastic reach-avoid set characterization, Proc. 50th IEEE CDC-ECC, pp. 7069-7074, 2011.

4) Yoshioka, H., Unami, K., and Fujihara, M. : Mathematical and numerical analyses on a Hamilton-Jacobi-Bellman equation governing ascending behaviour of fishes, RIMS Kôkyûroku, No. 1946, pp. 250-260, 2015.

5) Øksendal, B.: Stochastic Differential Equations, Springer-Verlag, Berlin, 2007.

6) Boulbrachene, M. and Haiour, M. : The Finite element approximation of Hamilton-Jacobi-Bellman equations, Comput. Math. Appl., Vol. 41, No. 7-8, pp. 993-1007, 2001.

7) Cai, L., Xie, W., Nie, Y., and Feng, J. : High-resolution semi-discrete Hermite central-upwind scheme for multidimensional Hamilton-Jacobi equations, Appl. Numer. Math., Vol. 80, pp. 22-45, 2014.

8) Govindarajana, N., De Visser, C.C., and Krishnakumar, K. : A sparse collocation method for solving time-dependent HJB equations using multivariate B-splines, Automatica, Vol. 50, No. 9, pp. 2234-2244, 2014.

9) Cristiani, E. and Falcone, M. : Fast semi-Lagrangian schemes for the Eikonal equation and applications, SIAM J. Numer. Anal., Vol. 45, No. 5, pp. 1979-2011, 2007.

10) Falcone, M. and Ferretti, R. : Semi-Lagrangian schemes for Hamilton-Jacobi equations, discrete representation formulae and Godunov methods, J. Comput. Phys., Vol. 175, No. 2 , pp. 559-575, 2002.

11) Barth, T.J. and Sethian, J.A. : Numerical schemes for the Hamilton-Jacobi and level set equations on triangulated domains, J. Comput. Phys., Vol. 145, No. 1, pp. 1-40, 1998

12) Boulaaras, S. and Haiour, M. : The theta time scheme combined with a finite-element spatial approximation in the evolutionary Hamilton-Jacobi-Bellman equation with linear source terms, Comput. Math. Model., Vol. 25, No. 3, pp. 423-438, 2014.

13) Horváth, T.L. and Mincsovics, M.E. : Discrete maximum principle for interior penalty discontinuous Galerkin methods, Central Euro. J. Math., Vol. 11, No. 4, pp. 664-679, 2013.
14) Idelsohn, S., Nigro, N., Storti, M., and Buscaglia, G. : A Petrov-Galerkin formulation for advection-reaction -diffusion problems, Comput. Methods. Appl. Mech. Eng., Vol. 136, No. 1-2, pp. 27-46, 1996.

15) Chen, Q., Hong, Y., and Temam, R. : Analysis of a penalty method, J. Sci. Comput., Vol. 53, No. 1, pp. 3-34, 2012

16) Pop, I.S., Radu, F., and Knabner, P. : Mixed finite elements for the Richards' equation: linearization procedure, $J$. Comput. Appl. Math., Vol. 168, No. 1, pp. 365-373, 2004.

17) Radu, F.A., Nordbotten, J.M., Pop, I.S., and Kumar, K. : A robust linearization scheme for finite volume based discretizations for simulation of two-phase flow in porous media, J. Comput. Appl. Math., Vol. 289, pp. 134-141, 2015.

18) Monterio, I.O., Manica, C., and Rebholz, L.G. : Numerical study of a regularized barotropic vorticity model of geophysical flow, Numer. Methods Partial Differ. Equat., Vol. 31, No. 5, pp. 1492-1514, 2015.

19) Neda, M., Sun, X., and Yu, L. : Increasing accuracy and efficiency for regularized Navier-Stokes equations, Acta Appl. Math., Vol. 118, No. 1, pp. 57-79, 2012.

20) Zhang, K., Teo, K.L., and Swartz, M. : A robust numerical scheme for pricing American options under regime switching based on penalty method, Comput. Econ., Vol. 43 No. 4, pp. 463-483, 2014.

21) Zvan, R., Forsyth, P.A., and Vetzal, K.R. : Penalty methods for American options with stochastic volatility, J. Comput. Appl. Math., Vol. 91, No. 2, pp. 199-218, 1998.

22) Yoshioka, H., Unami, K., and Fujihara, M. : A conforming finite element scheme for Hamilton-Jacobi-Bellman equations defined on connected graphs, Proc. Computational Engineering Conference, Vol. 20, Paper No. F-9-3, pp. 1-6, 2015.

23) Toro, E.F.: Riemann Solvers and Numerical Methods for Fluid Dynamics, Springer, 2009.

24) Vazquez-Cendon, E., Hidalgo, A., Garcia-Navarro, P., and Cea, L. : Numerical Methods for Hyperbolic Equations, CRC Press, 2012.

25) Behrens, J. : Adaptive Atmospheric Modeling, Springer, 2006.

26) Schmidt, A. and Siebert, K.G. : Design of Adaptive Finite Element Software, Springer, 2005.

27) Huang, W. and Russel, R.D. : Adaptive Moving Mesh Methods, Springer New York Dordrecht Heidelberg London, pp. 27-133, 2011.

28) Soheili, A.R., Kerayechian, A., and Davoodi, N. : Adaptive numerical method for Burgers-type nonlinear equations, Appl. Math. Comput., Vol. 219, No. 8, pp. 3486-3495, 2012.

29) Tang, H.Z., Tang, T., and Zhang, P. : An adaptive mesh redistribution method for nonlinear Hamilton-Jacobi equations in two- and three-dimensions, J. Comput. Phys., Vol. 188, No. 2, pp. 543-572, 2003.

30) Dong, H., Qiao, Z., Sun, S., and Tang, T. : Adaptive moving grid methods for two-phase flow in porous media, $J$. Comput. Appl. Math., Vol. 265, pp. 139-150, 2014.

31) Felcman, J. and Kadrnka, L : Adaptive finite volume approximation of the shallow water equations, Appl. Math. Comput., Vol. 219, No. 7, pp. 3354-3366, 2012.

32) Cao, W., Huang, W., and Russell, R.D.: A study on monitor functions for two-dimensional adaptive mesh generation, SIAM J. Sci. Comput., Vol. 20, No. 6, pp. 1978-1994, 2006.

33) Remski, J., Zhang, J., and Du, Q.: On balanced moving mesh methods, J. Comput. Appl. Math., Vol. 265, pp. 255-263, 2014.

34) Yoshioka, H, Unami, K., and Fujihara, M. : Mathematical analysis on a conforming finite element scheme for advec- 
tion-dispersion-decay equations on connected graphs, $J$. JSCE A2, Vol. 70, No. 2, pp. I_265-I_276, 2014.

35) Yoshioka, Unami, K., and Fujihara, M. : A conforming finite element method for non-conservative advection-diffusion equations on connected graphs, J. JSCE A2, Vol. 69, No. 2, pp. I_59-I_70, 2013. (in Japanese)

36) Camilli, F., Festa, A., and Schieborn, D. : An approximation scheme for a Hamilton-Jacobi equation defined on a network, Appl. Numer. Math., Vol. 73, pp. 33-47, 2013.

37) Lolla, T., Lermusiaux, P. F. J., Ueckermann, M. P. U., and Haley Jr., P. J. : Time-optimal path planning in dynamic flows using level set equations: theory and schemes, Ocean Dyn., Vol. 64, No. 10, pp. 1373-1397, 2014.

38) Yoshioka, H., Unami, K., Takagi, K., and Fujihara, M. : Application of a regime-switching diffusion process model to transport phenomena in surface water bodies, RIMS Kôkyûroku, No. 1952, pp. 55-62, 2015.

39) Yamamoto, T. and Oishi, S. : A mathematical theory for numerical treatment of nonlinear two-point boundary value problems, Jpn. J. Ind. Appl. Math., Vol. 23, No. 1, pp. 31-62.

40) Westerink, J. J. and Shea, D. : Consistent higher degree Petrov-Galerkin methods for the solution of the transient convection-diffusion equation, Int. J. Numer. Methods Engrg., Vol. 28, No. 4, pp. 1077-1101, 1989.

41) Shih, Y., Cheng, J. Y., and Chen, K. T. : An exponential-fitting finite element method for convection-diffusion problems, Appl. Math. Comput., Vol. 217, No. 12, pp. 5798-5809, 2011.

42) Rahman, M.M. and Siikonen, T. : An artificial compressibility method for viscous incompressibility and low Mach numbers, Int. J. Numer. Methods Engrg., Vol. 75, No. 11, pp. 1320-1340.
43) Hauke, G., Sangalli, G., and Doweidar, M.H. : Combining adjoint stabilization methods for the advection-diffusion-reaction problem, Math. Model. Methods Appl. Sci., Vol. 17, No. 2, pp. 305-326, 2007.

44) Nadukandi, P., Oñate, E., and Garcia, J. : A high-resolution Petrov-Galerkin method for the 1D convection-diffusion-reaction problem, Comput. Methods Appl. Mech. Engrg., Vol. 199, No. 9, pp. 525-546, 2011.

45) de Falco, C. and O'Riordan, E. : A parameter robust Petrov-Galerkin scheme for advection-diffusion-reaction equations, Numer. Algor., Vol. 56, No. 1, pp. 107-127, 2011.

46) O'Riordan, E. and Stynes, M. : An analysis of a superconvergence result for a singularly perturbed boundary value problem, Math. Comput., Vol. 46, No. 173, pp. 81-92, 1986.

47) Yaegashi, Y., Yoshioka, H., Unami, K., and Fujihara, M. : Conservative and adaptive numerical schemes for Kolmogorov's forward equations in unbounded 1-D domains, Proc. 18th Symposium on Applied Mechanics, No. 100022, 2015.

48) Lukas, P. : Optimization of parameters in SDFEM for different spaces of parameters, Appl. Math. Comp. DOI: 10.1016/j.amc.2014.12.084. (in press)

49) Takagi, K., Yoshioka, H., Unami, K., and Fujihara, M. : Conservative and adaptive numerical schemes for Kolmogorov's forward equations in unbounded 1-D domains, Proc. 18th Symposium on Applied Mechanics, No. 100051, 2015.

(Received June 23, 2015) 